
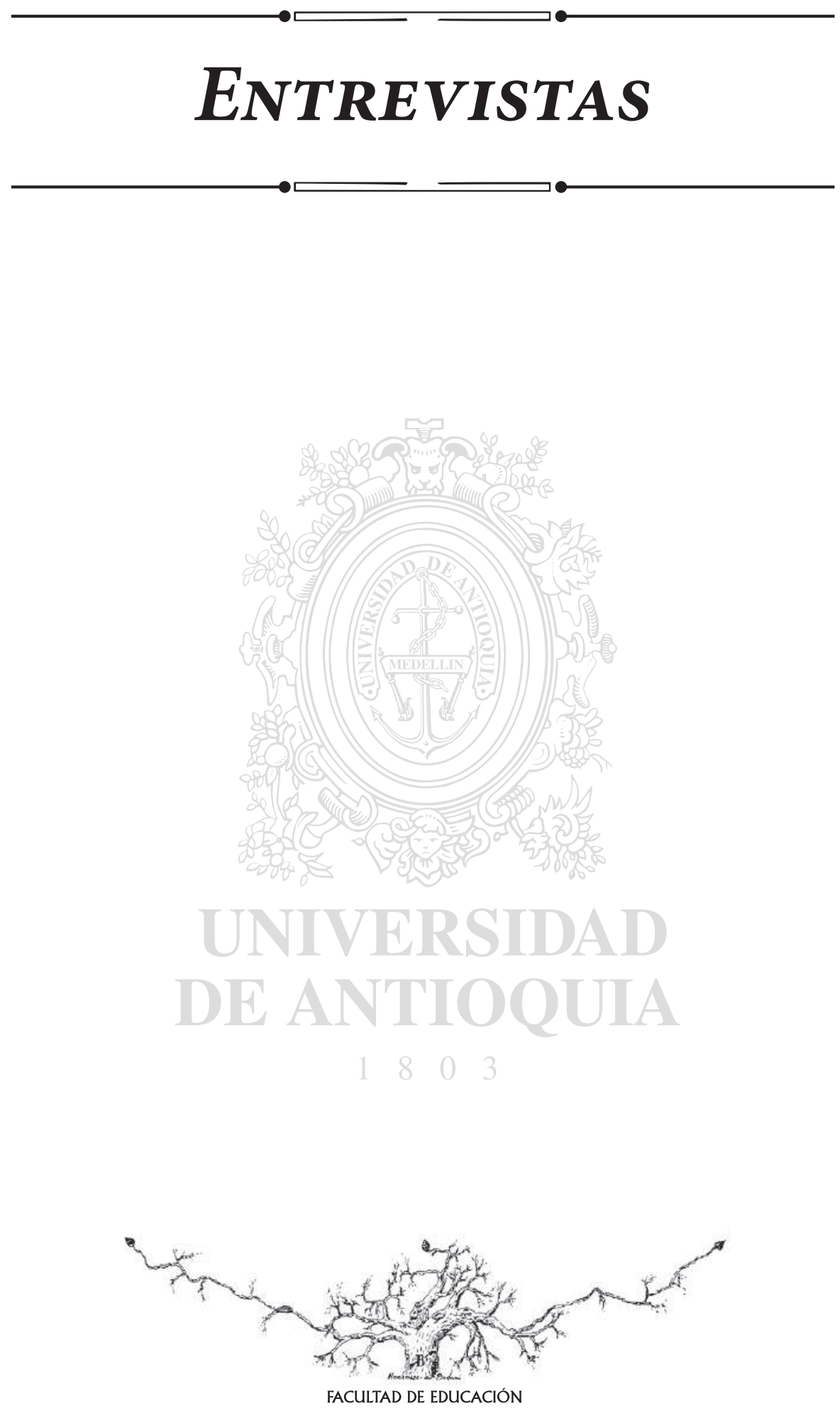


\title{
Formación en Ciencias Sociales y de los futuros profesores. Un diálogo con el profesor Joan Pagés Blanch
}

\author{
Education in Social Science and of Prospective Teachers. \\ Interview with the Professor Joan Pagés Blanch
}

\author{
Ruth Elena Quiroz Posada* \\ (iD) https://orcid.org/0000-0003-2726-8288 \\ Jhony Alexander Villa-Ochoa ${ }^{* *}$ \\ (iD) https://orcid.org/0000-0003-2950-1362
}

Tipo de Artículo: Entrevistas

Doi: 10.17533/udea.unipluri.19.1.06

Cómo citar este artículo:

Quiroz Posada, R. E y Villa-Ochoa, J.A. (2019). Formación en Ciencias Sociales y de los futuros profesores Un diálogo con el profesor Joan Pagés Blanch. Uni-pluriversidad, 19(1) , 87-96.Doi: 10.17533/udea.unipluri.19.1.06

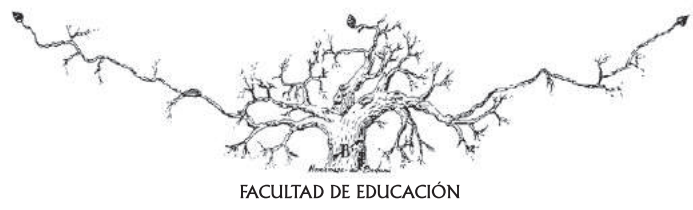

Recibido: 2019-08-10 • Aprobado: 2019-09-05

* Profesora Titular, Facultad de Educación, Universidad de Antioquia.

E-mail: ruth.quiroz@udea.edu.co

** Profesor Titular, Facultad de Educación, Universidad de Antioquia. Director de la Revista Uni-pluriversidad. E-mail: jhony.villa@udea.edu.co 


\title{
Resumen
}

Este artículo presenta algunas ideas del profesor Joan Pagés acerca de la Didáctica de las Ciencias Sociales. El documento contiene comentarios del profesor relacionados con la importancia de trabajar en una didáctica basada en la resolución de problemas sociales, que ayude a los estudiantes a construir conocimientos y procedimientos y, a su vez, favorezca otras alternativas críticas sobre la realidad social, así como el desarrollo de habilidades que organicen y seleccionen la información más pertinente para proponer soluciones. De ahí la importancia de cualificar la formación de los futuros profesores.

Palabras clave: formación del profesorado, didáctica de las Ciencias sociales, resolución de problemas sociales.

\begin{abstract}
This article presents some ideas about Social Sciences Teaching by the Professor Joan Pagés. The document contains his comments in relation to the importance of working on a teaching based on solving social problems. It helps students to build different knowledge and procedures and promotes also other critical alternatives on social reality, as well as the development of skills in order to organize and select the most appropriate information to propose solutions. Hence, the importance of qualifying the training of prospective teachers.
\end{abstract}

Keywords: Teacher Training, Social Sciences Teaching, Solving Social Problems. 


\section{INTRODUCCIÓN}

口-

La formación de profesores en las distintas áreas del conocimiento se ha consolidado como un área de investigación en la que confluyen diversos enfoques, intereses y perspectivas teóricas. Por ejemplo, en la búsqueda de estrategias para la formación de profesores en humanidades, Pérez Guzmán (2018) utilizó las "experiencias auténticas de lectura", es decir, espacios que permiten a los futuros profesores la posibilidad de experimentar "otras formas posibles de abordar la lectura, la escritura y la oralidad en la escuela" (p.27), partiendo de un contexto concreto, de una situación real. Por su parte, Betancur, Vásquez Yepes y Vanegas Hurtado (2018) sugieren que los profesores de lenguaje deben tener experiencias en el desarrollo de prácticas socioculturales. Para ellos, por ejemplo, una comprensión de la lectura y la escritura necesariamente debe considerar el mundo de la digitalidad y las necesidades de formación respectivas.
En el ámbito de la didáctica de las Ciencias Sociales, Pagés (1994) señala que "como la del resto de asignaturas, ha crecido y se ha amparado en las concepciones curriculares dominantes en cada momento histórico" (p.38) y, por tanto, es conveniente "prestar atención a las posibilidades que ofrecen los currículos prescritos para mantener la actual situación de la enseñanza de la historia y las ciencias sociales o para intentar cambiarla y, a su vez, mantener o cambiar la formación del profesorado responsable de su implementación en la práctica" (p. 39).

Con motivo de la visita del profesor Pagés a la Universidad de Antioquia en julio de 2019 se preparó un diálogo relacionado con sus aportes a la Didáctica de las Ciencias Sociales y a la formación de profesores. A partir de esta entrevista, se elaboró este artículo en el cual se presentan algunas ideas del profesor frente al tema y la importancia de trabajar en una didáctica basada en la resolución de problemas sociales.

\section{El diálogo}

Ruth Quiroz: Joan, manifestamos nuestro agradecimiento por estar en la Universidad de Antioquia y por aceptar esta entrevista. Eres nuestro amigo incondicional, colega universitario y excelente académico en los campos de la Didáctica de la Historia y de las Ciencias Sociales, la formación del profesorado y, por supuesto, la Educación. Queremos aprovechar tu visita para hablar acerca de tus visiones y avances en estos temas. Para comenzar quisiera que nos contextualizaras: ¿Quién es Joan Pagés y cuál es la obra que está haciendo?

Joan Pagés: ¡Es una pregunta de alto valor epistémico! ¡Es broma! Yo diría que soy una persona que ha tenido suerte en la vida porque he podido hacer lo que me ha gustado; ahora mismo estoy acá en Medellín haciendo lo que me gusta, y lo que me gusta es formar profesores, intercambiar con profesores conocimientos para pensar conjuntamente la educación como el pilar fundamental en el desarrollo del país y del mundo. Hoy, mi interés está centrado en intentar convencer a las generaciones que me van a sustituir de que vale la pena hacer un esfuerzo y un sacrificio para dignificar la profesión docente, para "salvar las escuelas" en la medida que son salvables en una sociedad en la que la comunicación y la información ya 
no pasan exclusivamente por la escuela sino por otros medios. Es necesario seguir creyendo y pensando en que la especie humana, si se educa, es una especie capaz de mejorar cualitativamente las condiciones de vida de este mundo y salvarlo y salvarse de los peligros que está sufriendo a causa del egoísmo de unos pocos que, a costa de enriquecerse, quieren acabar con el bienestar de todos.

Ruth Quiroz: ¿cuál dirías que ha sido tu mayor logro y tu mayor dificultad?

Joan Pagés: mi mayor logro ha sido conseguir, a través de la construcción de grupos, redes y comunidades académicas el hacer creíble la didáctica, como un campo de conocimiento universitario. Sé que esto no es exclusivamente un mérito mío; pero sí que creo que con otra gente hemos conseguido dignificar la didáctica y hacerla creíble. Seguramente, mi debilidad ha sido que no he conseguido hacer una buena transmisión a las generaciones del futuro. El problema que ahora tenemos es que profesores con mucha experiencia, que han investigado en el campo y que han hecho sus tesis doctorales, no pueden ser profesores universitarios, y no porque no conozcan la profesión sino porque no han publicado en una revista indexada, como si esto fuera lo más importante. Mi mayor preocupación es que no hemos sabido crear las condiciones para que aquellos docentes que tienen mucha experiencia, que han reflexionado, que han investigado, que son profesores de básica o de media, no puedan ser profesores universitarios y participar en la formación de las nuevas generaciones de docentes. La participación de los $\mathrm{y}$ de las docentes es fundamental en la construcción del campo.

Ruth Quiroz: sí, es verdad, ello es lamentable.
Joan Pagés: os pongo un ejemplo de un colega de mi Universidad, Joan Llusà. Un profesor al que formé como maestro, que trabajó en la enseñanza primaria, que luego hizo su Licenciatura en Historia, trabajó y sigue trabajando en la enseñanza secundaria, hizo su maestria y su tesis en el Doctorado en Educación, ha realizado pasantías en Chile y Argentina pero no lo han acreditado para poder optar a una plaza de profesor en didáctica de las ciencias sociales después de treinta años trabajando. ¿La razón? No ha publicado un artículo en inglés en una revista indexada ¡Es increíble! En cambio, un jovencito que no ha pisado un aula puede ser profesor porque tiene un artículo o dos en una revista indexada. Esto, creo, es un problema grave, luchamos mucho por conseguir un cuerpo único de enseñantes, pero hemos acabado con un cuerpo de enseñantes fragmentados en función de criterios políticos, que no responde a necesidades, contextuales, sociales o académicas.

Ruth Quiroz: ¿por qué trabajar con los profesores y en especial por qué ayudarnos a construir redes nacionales e iberoamericanas de grupos de investigación en Didáctica de las Ciencias Sociales? ¿Cuál es tu finalidad?

Joan Pagés: tengo la suerte de que me hice profesor en la práctica y mi modelo de formación fue un modelo de trabajo cooperativo. El hecho de participar en un movimiento de renovación pedagógica, del que aún soy miembro, me dio un sentido diferente de la docencia al de que aquellos que recibían una formación universitaria. En la universidad no me enseñaron a enseñar. Por tanto, yo me estrellé cuando empecé a trabajar en escuela porque nadie me había dado referencias de ningún tipo. Pero esto generó un aprendizaje que luego me ha parecido relevante, como me parece relevante lo que he 
logrado resignificar en las relaciones entre la investigación, la práctica docente y la formación del profesorado.

Los problemas de la práctica docente son universales, son globales. Por consiguiente, yo puedo aprender de lo que ocurre en Colombia y un colombiano puede aprender de lo que ocurre en Europa. En la medida en que seamos capaces de crear sinergias y de crear un modelo global de formación de profesores, estaremos preparándonos para hacer frente al reto que supone a formacion del profesorado del siglo XXI.

En mi opinión, los contenidos sociales, geográficos e históticos que se enseñan en prácticamente todo el mundo aún siguen claramente decantados por socializar a los y a las jóvenes ciudadanos en los valores derivados del estado nación. Estos contenidos ya no sirven ni siquiera para construir tu propia identidad y, desde luego, no sirven para comprender el mundo, las relaciones entre lo local y lo global y los problemas emergentes que exigen soluciones urgentes. Los contenidos que han de enseñar los y las docentes han de ayudar al alumnado a pensar críticamente y han de poner las bases para una cultura escolar contrahegemónica. La escuela ha de enseñar a pensar el mundo, a construir ideas propias sobre él y sobre la manera de actuar, de participar en él. Es probable que un pensamiento así construido no sea coincidente con las ideas de los gobiernos de turno. Esto es muy democrático en una sociedad diversa y plural, pero es peligroso para el sistema imperante. Mi idea de trabajar con los profesores de América Latina es que considero que aprendo de ustedes y espero poder enseñarles algo, además de fomentar la solidaridad, el trabajo en red y pensar desde un mundo global. No sé si seremos capaces de construir una -redinternacional docente. Yo desde luego no lo veré ya como docente.
A mis alumnos les digo: miren, hoy parece que solo el mundo es global económicamente, pero con la Revolución Industrial el movimiento obrero lo primero que hizo fue crear la Asociación Internacional de los Trabajadores o Primera Internacional, fundada en Londres. Su fin era la organización política del proletariado en Europa y el resto del mundo. Abrieron un foro para examinar problemas en común y proponer líneas de acción porque entendían que los problemas del capital eran globales y dijeron algo tan simple como " ¡Agrupémonos todos en la lucha final del género humano es la consigna internacional!” ¿Por qué hemos perdido de vista ese referente? Al margen de que luego hubo peleas y de la Primera se pasó a la Segunda, de la Segunda se pasó a la Tercera y de la Tercera a no sé cuántas Cuartas. Siempre tendremos dificultades y desencuentros porque así somos los seres humanos. El diálogo ayudará a resolverolos. Pero ello no es razón para no intentar ponernos de acuerdo cuando los problemas son comunes. Y los problemas de la enseñanza de las ciencias sociales, de la geografía, de la historia y de la formación ciudadana son comunes en todo el mundo. Por esta razón creo que es fundamental organizarnos y dar a conocer nuestro trabajo.

Es preciso defender la idea de un mundo global también desde la perspectiva de la educación, de que el ser humano tiene los mismos problemas y necesidades. Sabemos que los contextos, especialmente los sociales son distintos, pero las respuestas a estos problemas siempre serán más culturales que nacionales y nos ayudarán a defender la diversidad dentro del más absoluto respeto por la dignidad humana.

Ruth Quiroz: desde tu experiencia ¿Cuáles serían los nuevos retos de la Didáctica de las Ciencias Sociales? 
Joan Pagés: la Didáctica de las Ciencias Sociales es una disciplina joven, faltan muchas más investigaciones y su corpus teórico y conceptual está en evolución. Falta una mejor formación en los profesores que ya ejercen y una preparación más clara de los que se forman para que puedan ejercer como prácticos reflexivos e intelectuales comprometidos.

Las modalidades más frecuentes de formación continuada del profesorado suelen ubicarse aún dentro del modelo curricular técnico y se caracterizan, en el caso de la Didáctica de las Ciencias Sociales, por tener un carácter más informativo que formativo, y por poner más el énfasis en lo que debería hacer que en lo que ya está haciendo y en los problemas que realmente tiene. Es fundamental que los y las docentes sepan qué racionalidad mueve sus prácticas. Sin embargo, en la formación no se establecen con facilidad puentes entre la teoría y la práctica, no se ofrecen conocimientos teóricos desde la práctica ni tampoco conocimientos prácticos desde la teoría.

El reto fundamental de la Didáctica de las Ciencias Sociales es reflexionar y buscar alternativas sobre el uso social del conocimiento geográfico, histórico y social hoy. Considero que ahí hay un abismo entre lo que estamos investigando, enseñando y el mundo. En el caso de nuestra área está clarísimo ¿Cómo explicamos los fenómenos que ocurren en el mundo? ¿Cómo explicamos el brote del fascismo? ¿Cómo explicamos la pervivencia del machismo? ¿Cómo explicamos la existencia de la violencia de género? ¿Cómo explicamos la destrucción del medio ambiente? ¿Cómo explicamos el incremento de la siniestralidad? Un buen conocimiento escolar centrado en los problemas reales, en problemas sociales no nos permitiría perdernos. Creo que este es el reto ¿cómo cons- truimos un currículum que obedezca a los problemas sociales relevantes y aquello que a la gente le pasa realmente en la vida? No solamente en ciencias sociales sino también en ciencias de la salud, en ciencias ambientales, en ciencias físicas, en todo. En el uso social del lenguaje y de las matemáticas.

Algo hacemos mal en las aulas de clase o no lo hacemos como deberíamos hacerlo. Me preocupa la deriva electoral que se está produciendo en el mundo. No parece que se elija a los y a las mejores para dirigir nuestros países si no a quienes más gritan y mejor manejan twitter. Hoy cuando estoy ya finalizando mi vida profesional, considero que uno de los puntos débiles de mi práctica es no haber conseguido que el $100 \%$ de los docentes que formé sean prácticos, reflexivos -¡una utopía, ya sé!- que eduquen a los y a las jóvenes ciudadanas para posicionarse crítica y creativamente ante los problemas del mundo y asuman su protagonismo con el orgullo de creer que otro mundo es posible.

Jhony A. Villa: como usted lo dijo, hay una necesidad de fundamentar la enseñanza y el aprendizaje de las Ciencias Sociales en problemas reales. Al respecto, usted mismo se pregunta ¿Cómo comprender cada uno de los aspectos anteriormente mencionados? Yo soy del área de Educación Matemática y nos preguntamos también frente a cuestiones como cuál es el sentido que tiene la Educación Matemática $\mathrm{y}$, en general, la ciencia frente a los problemas de la sociedad; los llamados problemas "reales". Dentro de su experiencia en la formación de profesores ¿Qué principios, prácticas o ideas estructuran un currículo para que atienda a esa necesidad de los problemas "reales"?

Joan Pagés: una clave principal es entender que las disciplinas escolares son medios y no fines en sí mismos. Yo no puedo 
pensar que mis estudiantes sean historiadores porque me equivocaría. Mis estudiantes necesitan saber suficiente historia para entender la historicidad del presente, para saber de dónde vienen, dónde están y por qué están donde están y hacia dónde quieren ir. Pero esto no les habilita como historiadores, sino que les permite ubicarse en su mundo y tomar decisiones conscientes en él y lo mismo diría en matemáticas y en cualquier otra disciplina. Esto me exige un cambio. He de pensar un contenido que se centre en problemas y no en conocimientos disciplinares organizados en parcelas. Problemas de ámbito social como ¿Por qué los afrocolombianos no están representados en el curriculum de historia o por qué están infrarrepresentados? ¿Por qué la movilidad en las ciudades está llegando al colapso? ¿Por qué a medida que vamos construyendo en la montaña destruimos el patrimonio natural? Todos estos son contenidos realmente interesantes, mucho más que estudiar la colonia, estudiar la prehistoria o estudiar el mundo clásico; temas que no propongo dejar de lado sino al contrario. Si centramos la enseñanza en problemas sociales relevantes, uno podría estudiar, por ejemplo, cuál era la relación entre el ser humano con el medio, la sociedad con el medio en el mundo clásico, en Grecia o en Roma, en el mundo medieval, en la colonia y hoy. Es decir, problemas humanos de ayer, hoy y mañana.

Se puede hacer comparaciones muy ricas y se puede comprobar cómo el ser humano progresivamente, a medida que ha "avanzado", ha ido destruyendo la naturaleza pero también ha descubierto medicinas para paliar y curar problemas de la salud o ha hecho posible mejorar las condiciones de vida. Es necesario enseñar conocimientos sociales críticos para frenar la destrucción y para proteger y conservar aquello que nos ha permitido evolucionar y llegar a cotas de bienestar como el derecho a la salud, a la educación, al trabajo y a la vivienda, cosas a las que no deberíamos renunciar. Hay una cosa en la que insisto mucho a mis alumnos: han de enseñar a los chicos y a las chicas que todo el mundo ríe, llora, nace, muere y se alimenta. Afortunadamente, como tenemos cultura, no reímos por las mismas cosas, no lloramos por las mismas cosas, nos alimentamos de cosas distintas, todo esto es cultura; y la cultura es lo esencial. La cultura no debe suponer discriminación para nadie: los afrocolombianos tienen su cultura, los indígenas tienen su cultura, los blancos de origen italiano o los de origen ucraniano tienen la suya y esto es riqueza; esto es cultura. Por ello, no hemos de compartir un estándar de ciudadanía, porque no existe, como no existe un modelo único de mujer o de hombre, somos hombres y mujeres de muchas maneras. Hemos de compartir, esto sí, unos mismos derechos y unos mismos deberes.

Jhony Villa: quisiera unir la pregunta que usted presentaba sobre una didáctica fundamentada en problemas reales con la formación del profesorado ¿Qué características o qué aspectos particulares tendría una formación de profesores vinculada, situada o fundamentada en problemas reales?

Joan Pagés: es una nueva forma de asumir la formación profesional docente. Sabemos que las disciplinas no se han construido como un arte para disfrutar de ellas. La matemática, la historia, la física, la química, la música, cualquier disciplina se construye porque dan respuestas a problemas humanos.

En el campo del ocio, en el campo de la salud, en el campo de la convivencia, en el campo del medio ambiente, en cualquier campo hay que llevar la teoría a la práctica, 
y desde la práctica hay que construir teoría y eso es relativamente sencillo. No es fácil construir un currículo basado en problemas reales ¿Hace falta un currículo crítico ordenado como el currículum técnico en un mundo en el que los cambios son continuos? Creo que no. Hay que preparar a los futuros docentes con conocimientos disciplinares basados en aquellas teorías, en aquellos conceptos y en aquellos procedimientos más relevantes de las mismas, aplicables a cualquier situación del pasado, del presente $\mathrm{y}$ del futuro. Los y las docentes han de saber transferir aquello que han aprendido en la universidad a los problemas que interesan a sus estudiantes y a la sociedad: problemas ambientales, problemas de convivencia, problemas de movilidad, problemas de machismo, problemas de chovinismo. Han de saber muy bien lo que han de enseñar pero no han de pretender imponer lo que saben a su alumnado, sino que han de saber adaptarlo a los propósitos del momento y del grupo de alumnos con que trabajen.

Por ejemplo, el actual problema de Venezuela y las migraciones de venezolanos a Colombia: ¿Por qué se da?, ¿Por qué han llegado tantos venezolanos a Colombia?, ¿Por qué han aparecido brotes de chovinismo y se está acusando a los venezolanos de muchos de los problemas y de los conflictos de la sociedad colombiana? Siempre me han preocupado los problemas derivados del chovinismo y del nacionalismo más radical. Allá y acá. Recuerdo que en uno de mis primeros viajes a Caracas, trabajando también en formación docente, apareció en clase un problema relacionado con las relaciones entre las personas que viven en Colombia y las que viven en Venezuela. Alguien dijo: "los colombianos matan"," los colombianos no son buenos vecinos" y uno que otro de estos estereotipos que se han construido con la intención de dividir, de separar. Yo les dije: ¿Qué me dicen? ¿Qué diferencia hay entre el colombiano y el venezolano? Me parece que no hay muchas diferencias, que es más lo que les une que lo que les separa. Las diferencias entre ustedes, los venezolanos y los colombianos que las personas de origen europeo. Son mucho menores las diferencias entre ustedes y las poblaciones indigenas que habitan en sus países y que no siempre se sienten ni colombianas ni venezolanas. ¿Qué quiero decirles con ello?

Estamos hablando de Venezuela o Colombia, hablamos de un constructo creado a raiz de las independencias que interesaba fundamentalmente a las burguesías nacionales que crearon fronteras y nuevos países. No estamos hablando de problemas de minorías étnicas que ocupaban este territorio antes de la venida de los europeos; estamos hablando de la clase dirigente criolla que se independizó para explotar más y mejor los bienes naturales de cada territorio, y el caso más evidente no es ni siquiera Colombia y Venezuela, sino Panamá, Costa Rica, Nicaragua, Salvador, Honduras, Guatemala, es decir Centroamérica. La fragmentación de un territorio ocupado anteriormente por poblaciones indígenas sin fronteras. Las fronteras están ahí, en todo el mundo ¿Por qué existen?, ¿desde cuándo existen? Hoy las fronteras separan fundamentalmente personas. No separan mercancias, dinero, drogas y muchas otras cosas que benefician a las minorías dirigentes de nuestros países y a quienes realmente dirigen el mundo.

Trabajamos con mis alumnos la evolución de Europa, la creación de la Unión Europea. Los europeos han dejado de matarse desde que los alemanes y los franceses se sentaron y decidieron que se acabaran las disputas y desaparecieran las fronteras. En la comunidad europea ya no hay fronteras 
o si se requieren es para personas que vienen de otras partes, pero cuando tú estás en Europa puedes recorrer desde el Sur de España hasta el Norte de Suecia sin encontrar una sola frontera y sin que nadie te pida la documentación. Pero de repente, apareció el brexit y los intereses económicos de determinadas burguesias nacionales quieren deshacer aquello que no los beneficia suficientemente. Son ejemplos de problemas que la escuela no puede, ni debe, obviar.

El currículo crítico concede importancia a las aportaciones disciplinares como soporte, tanto para la construcción de conocimientos, como para el análisis de los problemas sociales reales. Plantea el carácter ideológico del currículo y de la práctica. No hace falta un temario tan detallado y minucioso como el actual si los y las docentes tienen una buena formación universitaria, si yo sé lo que es la historia, si yo sé lo que es la matemática y cómo he de aplicar los conceptos, los procedimientos y las habilidades para entender los problemas del mundo. Los problemas están ahí. Trabajar problemas sociales en el aula es hacer una apuesta para que nuestro alumnado forme parte de una ciudadanía efectiva y participativa capaz de tomar decisiones argumentadas, críticas y propositivas. Este es el reto de la escuela del siglo XXI y de la enseñanza de las ciencias sociales, de la geografía y de la historia.

Ruth Quiroz: se trata de asumir los desafíos y buscar soluciones educativas de acuerdo con las necesidades, las prioridades y los contextos de los estudiantes. Además, tendrá que ser un trabajo colaborativo e interdisciplinario.

Joan Pagés: sí, efectivamente.

Ruth Quiroz y Jhony Villa: Profesor Joan, le damos las gracias por esta entrevista y por los aportes tan generosos que ha hecho frente al conocimiento y al trabajo colaborativo y en red que ha impulsado en la Facultad de Educación de la Universidad de Antioquia, desde hace ya varios años.

\section{CONSIDERACIONES FINALES}

En sus respuestas más relevantes, el profesor Pagés no genera modelizaciones de la Didáctica sino que plantea dudas, problemas y cuestiones a debatir y a seguir estudiando frente a los contenidos a ser enseñados, pero también frente a la formación del profesorado que debe enfrentar una realidad educativa difícil, llena de conflictos sociales y de comunidades aún por construir. Desde sus múltiples trabajos de investigación, el profesor Pagés nos lleva a reflexionar acerca de la enseñanza y posibilita mejorar la práctica pedagógica, ampliando el conocimiento de los profesores al ofrecerles inicialmente perspectivas complementarias a las propias y luego, a través de la divulgación de los resultados de otros informes o proyectos que él aporta para confrontar la práctica.

Al leer y escuchar al profesor Pagés siempre se evidencia coherencia entre "lo que dice y lo que hace", pues propone claves de transformación del conocimiento social en relación con el currículo, la investigación, la formación del profesorado y la enseñanza de los problemas sociales, políticos y económicos que nos aquejan en los planos global y local, incluyendo observaciones de sus continuos viajes. En las valoraciones realizadas por los profesores que le conocemos y que participamos de sus seminarios, 
diálogos y clases, lo reconocemos con admiración porque evidencia carisma en "el trato con el otro", respeto "por la cultura" y "gran conocimiento" que procede de la propia práctica, de sus experiencias como enseñante y de sus ideas sobre el mundo y la sociedad.

\section{REFERENCIAS}

Betancur, D., Vásquez Yepes, V. y Vanegas Hurtado, E. (2018). Una experiencia de formación inicial de maestros de lenguaje alrededor de la lectura y la escritura como prácticas socioculturales. Uni-pluriversidad, 18(1), 25-35. doi: https://doi.org/10.17533/udea.unipluri.18.1.03.

Pagés, J. (1994). La didáctica de las Ciencias Sociales, el currículum de Historia y la formación del profesorado. Signo, Teoría y Práctica de la Educación 13, 38-51. doi: https://historia1 imagen.files.wordpress.com/2011/10/pages-la-didactica-de-las-cs-sociales-el-curriculum-de-historia-y-la-formacion-de-profesorado.pdf.

Pérez Guzmán, J. (2018). La formación de maestros para la enseñanza del lenguaje como práctica social. Uni-pluriversidad, 18(1), 36-46. doi: https://doi.org/10.17533/udea.unipluri.18.1.04. 